\title{
Resistencia de los morteros de cemento a la corrosión producida por las disoluciones de sales magnésicas
}

(Comentarios al artículo publicado por W. Riedel en «Zement-Kalk-Cips», n.* 6, junio 1973)

PABLO GARCIA DE PAREDES Y CAIBROIS, Lcdo. en Ciencias Químicas

I.E.T.c.c.

Entre los agentes químicos que destruyen a los morteros y hormigones elaborados con conglomerantes hidráulicos, silícicos o aluminosos, inspira mucho temor el catión magnesio, principalmente si en las disoluciones acuosas le acompañan los aniones sulfato y/o cloruro. Por esta razón el trabajo del Sr. Riedel adquiere especial interés, tanto más cuánto no es numerosa ni resolutiva la experiencia expuesta en la literatura.

\section{N T R O D U C C I O N}

En ella el autor considera que el ensayo de la resistencia química realizado por la inmersión de probetas en disoluciones de sulfato sódico, de concentraciones al 5 y al $10 \%$, conduce a resultados erróneos, ya que, en la práctica, son otros los cationes que pueden existir, principalmente en los ambientes artificiales. Esta afirmación del Sr. Riedel confirma nuestra experiencia, reiteradamente comprobada en los laboratorios del I.E.T.c.c.; además son muchos otros los aniones destructores.

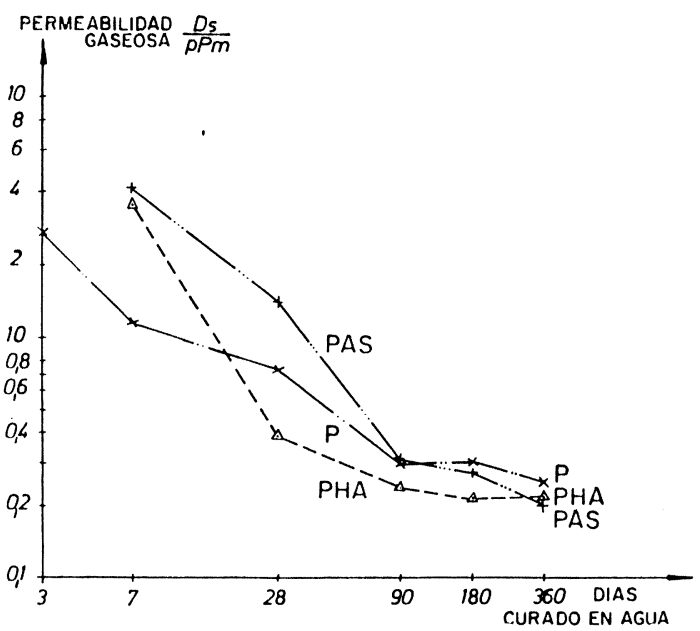

Fig. 1.-Permeabilidad a los gases de morteros normales según TCL 10573 , preparados con: portland 350. PAS 300 (Exento de $C_{3} A$ ). PHA 225. Desecados a $50^{\circ} \mathrm{C}$ y ensayados a $1,5 \mathrm{kp} / \mathrm{cm}^{2}$ de presión. 


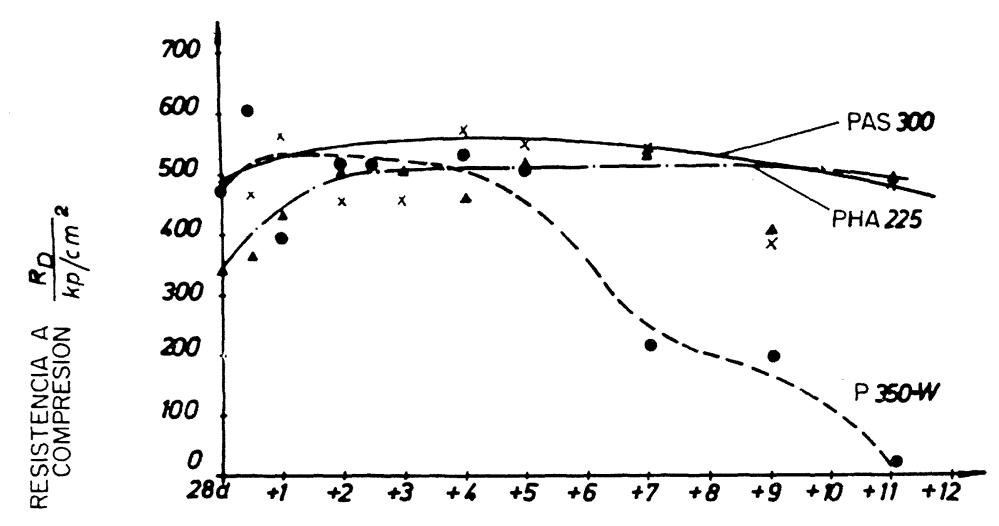

CURADO EN AGUA $1,269 \mathrm{MgSO}_{4} /$

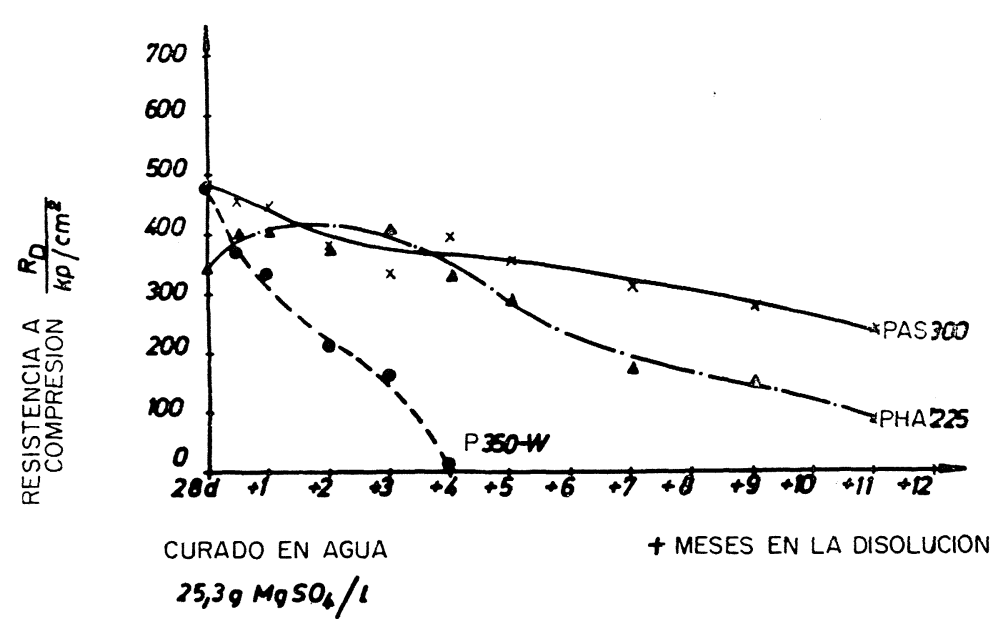

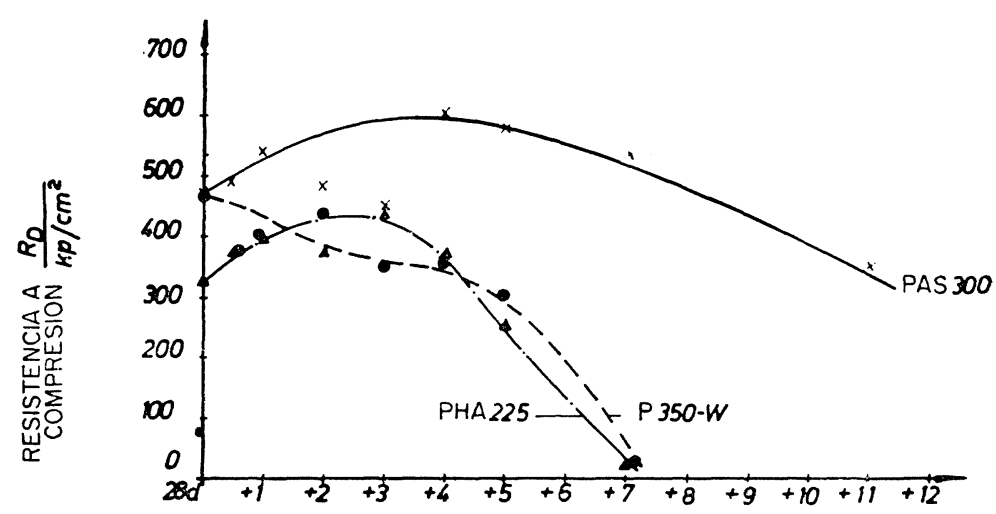

CURADO EN AGUA $126 \mathrm{~g} \mathrm{MgSO}_{4} / 6$

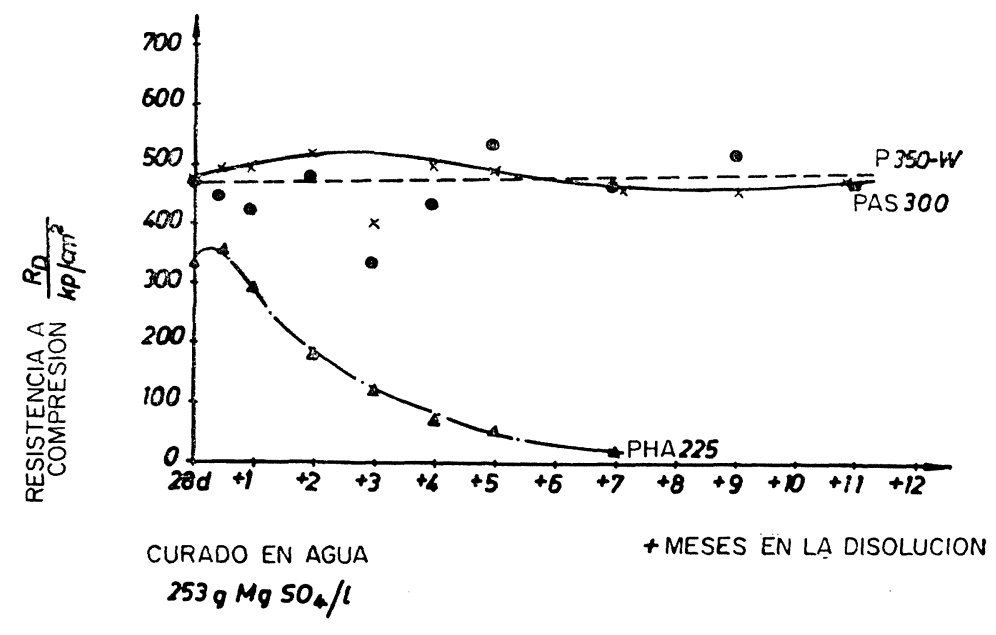

Fig. 2.-Resistencia a la compresión de prismas $10 \times 10 \times 60 \mathrm{~mm}^{3}$ preparados con P; PAS; PHA y conservados en disolución acuosa de $\mathrm{MgSO}_{4}$. 
Las experiencias llevadas a cabo por el ya citado Sr. Riedel, en la Escuela Superior de Arquitectura y Construcción de Weimar, tuvieron por objeto aportar conocimientos para recogerlos en las "Instrucciones" redactadas para el buen uso de los morteros y hormigones en obras relacionadas con las industrias de materiales potásicos en la República Democrática Alemana. Las disoluciones de sales potásicas, de esas industrias, contienen considerables cantidades de $\mathrm{MgCl}_{2}-\mathrm{MgSO}_{4}-\mathrm{KCl}-\mathrm{NaCl}-\mathrm{Na}_{2} \mathrm{SO}_{4}-\mathrm{K}_{2} \mathrm{SO}_{4}$ y muy poca cantidad de $\mathrm{CaSO}_{4}$. Generalmente el valor de $\mathrm{pH}$ oscila entre 6,2 y 8,5, es decir, son "industrialmente" "lejías".
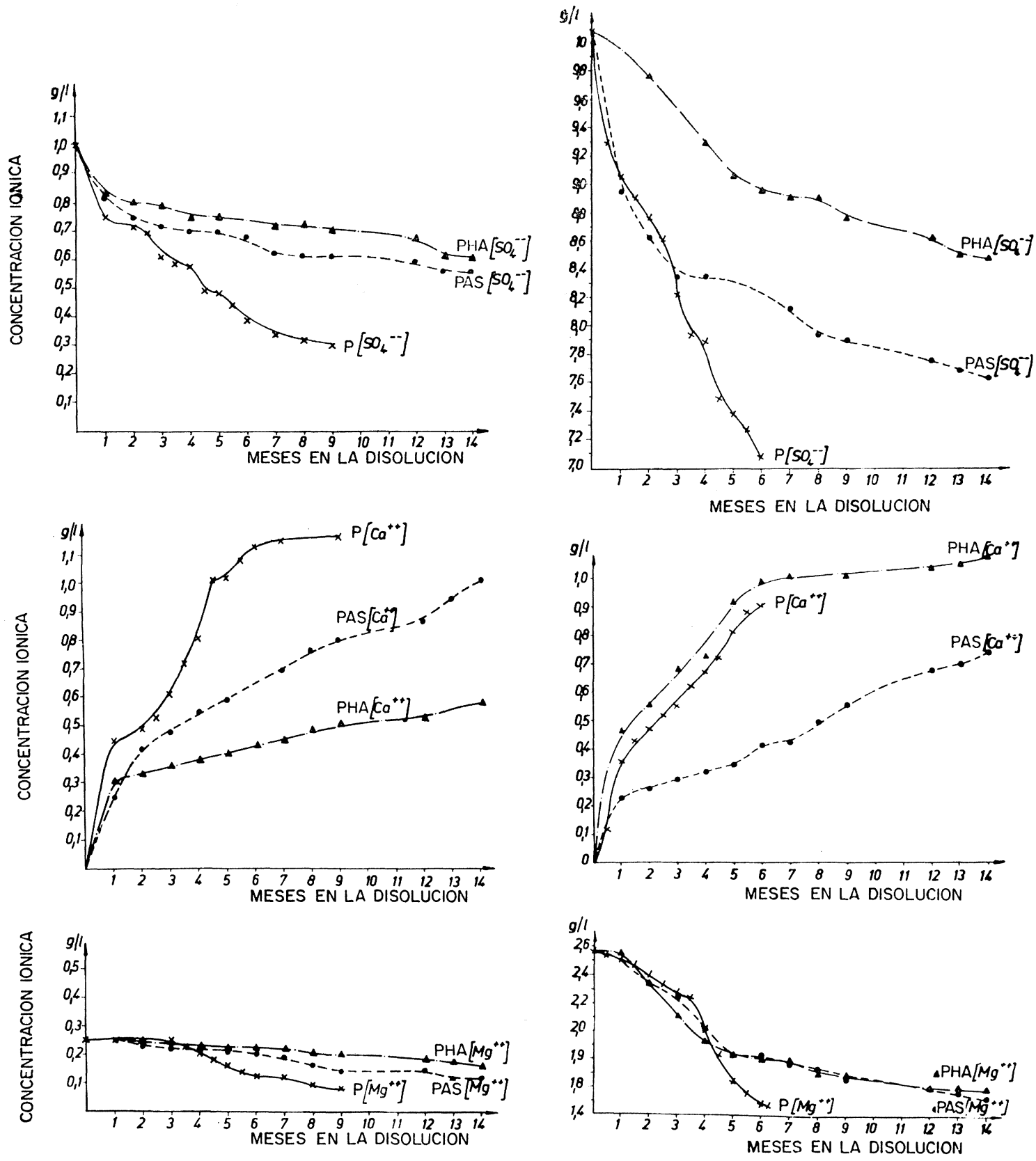

Fig. 3.-Variaciones en la concentración iónica de las disoluciones de sulfato magnésico durante el contacto con prismas de $10 \times 10 \times 60 \mathrm{~mm}^{3}$.

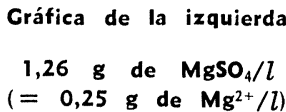

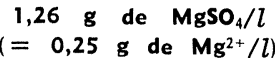

Gráfica de la derecha

$12,6 \mathrm{~g}$ de $\mathrm{MgSO}_{4} / l$

$\left(=2,55 \mathrm{~g}\right.$ de $\left.\mathrm{Mg}^{2+} / l\right)$ 


\section{Temas y planes de experimentación}

Para el autor la resistencia química de los morteros y hormigones depende, principalmente, del tipo de conglomerante utilizado y de la permeabilidad que el tipo de conglomerante y la técnica aplicada hayan producido.

La experimentación se hizo utilizando:

Portland $250 \mathrm{P}$

\begin{tabular}{|c|c|c|c|}
\hline $\mathbf{C}_{3} \mathrm{~A}$ & $\mathbf{C}_{4} \mathbf{A F}$ & $\mathrm{c}_{3} \mathrm{~S}$ & $\mathrm{C}_{2} \mathrm{~S}$ \\
\hline 13,8 & 8,6 & 52,8 & 17,5 \\
\hline 0,0 & 20,7 & 68,5 & 4,7 \\
\hline
\end{tabular}

Con ellos se prepararon prismas de mortero, según la norma TGL 10 573: 1 parte de cemento, 3 partes de arena. Dimensiones: $10 \times 10 \times 60 \mathrm{~mm}^{3}$.

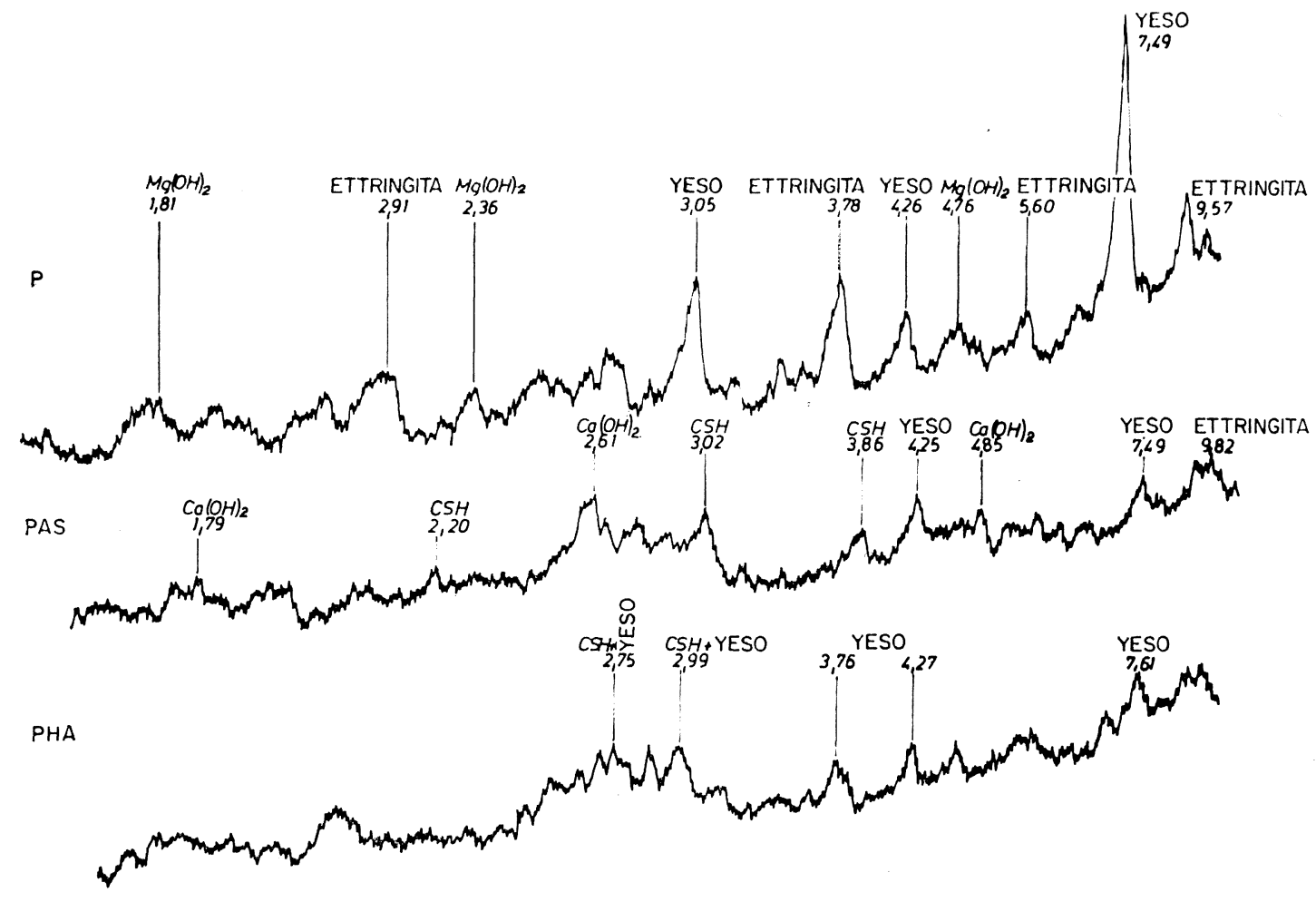

\footnotetext{
$\begin{array}{lllllllllllllllllllllllll}28 & 27 & 26 & 25 & 24 & 23 & 22 & 21 & 20 & 19 & 18 & 17 & 16 & 15 & 14 & 13 & 12 & 11 & 10 & 9 & 8 & 7 & 6 & 5\end{array}$

Fig. 4.-Difractogramas de rayọ $\mathrm{X}$. (Probetas sumergidas, durante 2 años, en disolución acuosa de $39 \mathrm{~g} \mathrm{MgSO}_{4} / l$.
}

Como agresivos, las disoluciones de sulfato magnésico y de cloruro magnésico con las siguientes concentraciones:

\begin{tabular}{crrr}
$\begin{array}{c}\mathbf{M g s O}_{4} \\
(\mathbf{g} / l)\end{array}$ & $\begin{array}{c}\mathbf{M g}^{2+} \\
(\mathbf{m g} / l)\end{array}$ & $\begin{array}{c}\mathbf{M g C l}_{\mathbf{2}} \\
(\mathbf{g} / l)\end{array}$ & $\begin{array}{c}\mathbf{M g}^{2+} \\
(\mathbf{m g} / l)\end{array}$ \\
\hline 1,26 & 255 & 1,0 & 255 \\
25,3 & 5.100 & 20,0 & 5.100 \\
126,0 & 25.500 & 100,0 & 25.500 \\
250,0 & 51.000 & 200,0 & 51.000
\end{tabular}


Las técnicas aplicadas fueron:

- Medida de la porosidad.

- Variación del módulo dinámico de elasticidad.

- Variación de la solidez, a través de las resistencias mecánicas.

- Variación de las concentraciones de las disoluciones agresivas.

- Modificaciones de la pasta pura, en prismas.

- Examen con rayos $\mathrm{X}$.

- Análisis térmico diferencial.

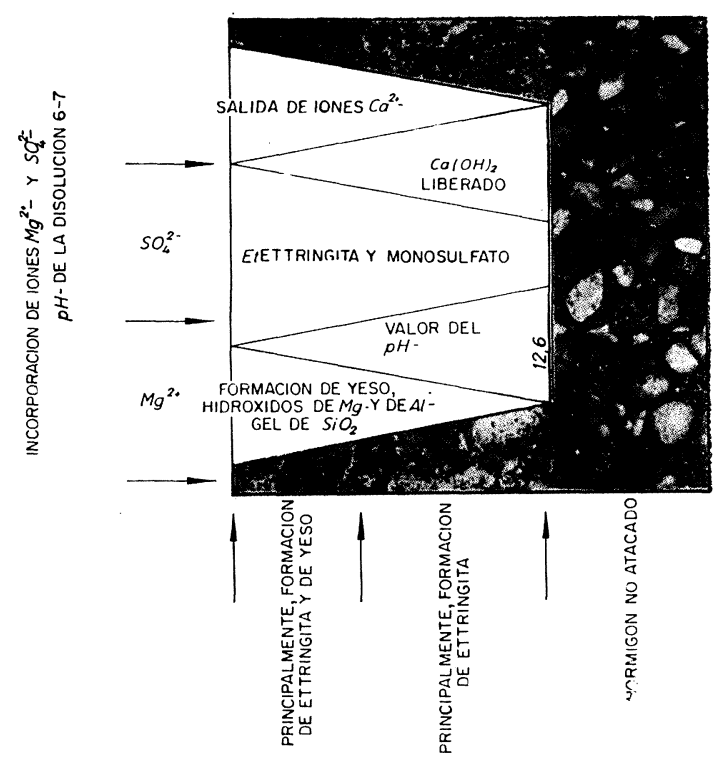

Fig. 5.-Modelo de corrosión por $\mathrm{MgSO}_{4}$. Disoluciones hasta $20 \mathrm{~g}$ de $\mathrm{MgSO}_{4} / l$.

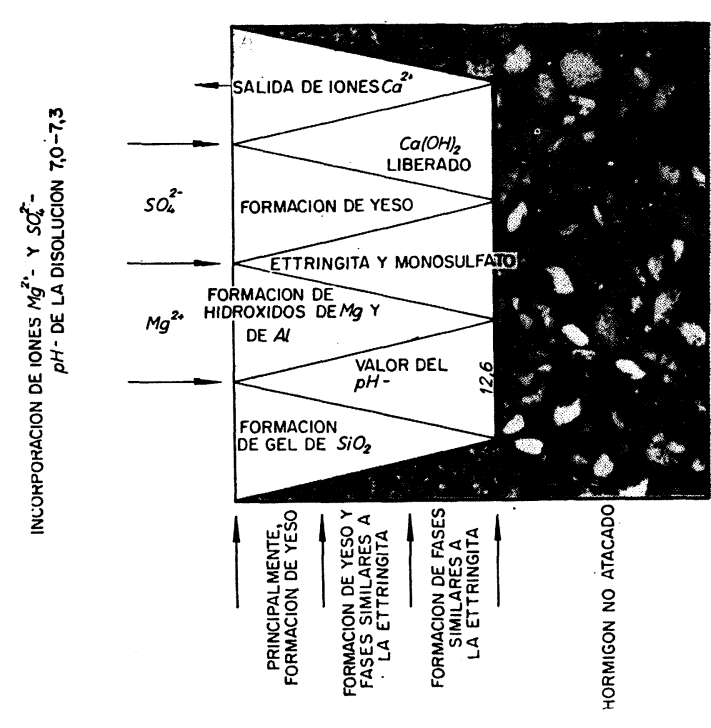

Fig. 6.-Modelo de corrosión por $\mathrm{MgSO}_{4}$. Disoluciones con 20 a $125 \mathrm{~g}$ de $\mathrm{MgSO}_{4} / l$.

\section{Resultados y comentarios}

Porosidad: El autor aplicó a las probetas, después de 28 días de conservación en las disoluciones agresivas, la medida de la penetrabilidad de una corriente de aire (Riedel, W. y Göhring, Ch. Wissenschaftliche Zeitschrift der Hochschule für Architektur und Bauwesen Weimar 1963, n. ${ }^{\circ}$ 6 655) y encontró notables diferencias entre los distintos morteros.

El cemento tipo PAS se hidrata con más lentitud que el tipo $\mathrm{P}$, pero a los 90 días la diferencia es muy pequeña y a los 360 días aparece más compacto el PAS. El cemento tipo PHA inicia muy lentamente su hidratación, pero a los 28 días es el que alcanza mayor compacidad.

Corrosión causada por el $\mathrm{MgSO}_{4}$ : Tanto las medidas del módulo elástico como los valores alcanzados por las resistencias mecánicas en los morteros sumergidos en las disoluciones agresivas, incrementan sus valores durante los primeros plazos de observación; esto demuestra que las combinaciones sulfáticas, primero, y más tarde, los nuevos com- 
puestos cristalinos rellenan los poros de la pasta cementicia. En los plazos sucesivos, el desarrollo de los cristales y sus exigencias espaciales originan una presión que da lugar a la fisuración, y finalmente, a la destrucción de los prismas.

El autor diferencia el proceso según el tipo de cemento:

Cemento portland P: en las disoluciones de $\mathrm{MgSO}_{4}$ de concentraciones hasta los 25,3 de $\mathrm{MgSO}_{4} / l$ posee muy pequeña resistencia química dependiente de la concentración de sulfato. Pero (esto es muy notable) en concentraciones muy altas, más de $250 \mathrm{~g}$ de $\mathrm{MgSO}_{4} / l$ aparecen los prismas de mortero de portland $\mathrm{P}$ tan resistentes como los de portland PAS. Esta resistencia química es independiente del contenido de aluminato tricálcico del portland.

Cemento portland PAS: hasta la concentración de $25,3 \mathrm{~g}$ de $\mathrm{MgSO}_{4} / l$ ofreció la mínima resistencia, (mis experiencias en el I.E.T.c.c. no permiten tan rotunda afirmación), pero en disoluciones mayores de $126 \mathrm{~g}$ de $\mathrm{MgSO}_{4} / l$ aumenta la resistencia.

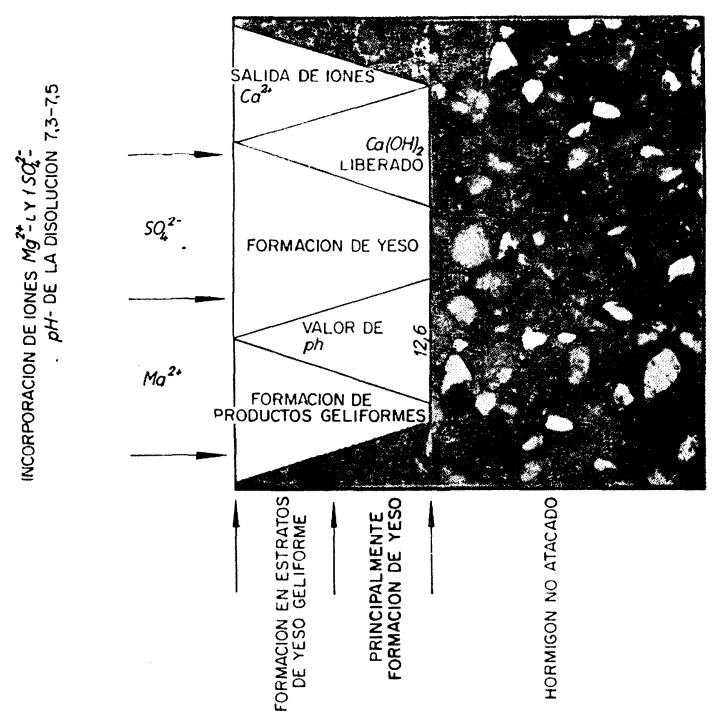

Fig. 7.-Modelo de corrosión por $\mathrm{MgSO}_{4}$. Disoluciones con más de $125 \mathrm{~g}$ de $\mathrm{MgSO}_{4} / l$.

Cemento de horno alto PHA: hasta las concentraciones de $20 \mathrm{~g}$ de $\mathrm{MgSO}_{4} / l$ su resistencia apenas se diferencia de la que posee el PAS. A concentraciones mayores pierde rápidamente resistencia química y es tanto más rápidamente destruido cuánto mayor es la concentración de sal magnésica.

A través de las variaciones que las probetas de mortero producen en el contenido iónico de las disoluciones agresivas durante el plazo de observación, el autor deduce las modificaciones que experimenta la resistencia química de los conglomerantes (fig. 3). En general, las disoluciones se enriquecen en cationes-calcio y se empobrecen en catión magnesio y en anión sulfato.

El cemento portland rico en aluminato tricálcico provoca estos cambios de concentración, más intensa y rápidamente que el exento de aluminato. La resistencia del cemento de horno alto en las disoluciones de $\mathrm{MgSO}_{4}$ de concentraciones hasta $20 \mathrm{~g} / l$ aparece relacionada con la pequeña disminución producida en la concentración iónica de $\mathrm{SO}_{4}{ }^{2-}$. Estas alteraciones de concentración coinciden muy bien con los resultados de los ensayos mecánicos (fig. 2). 

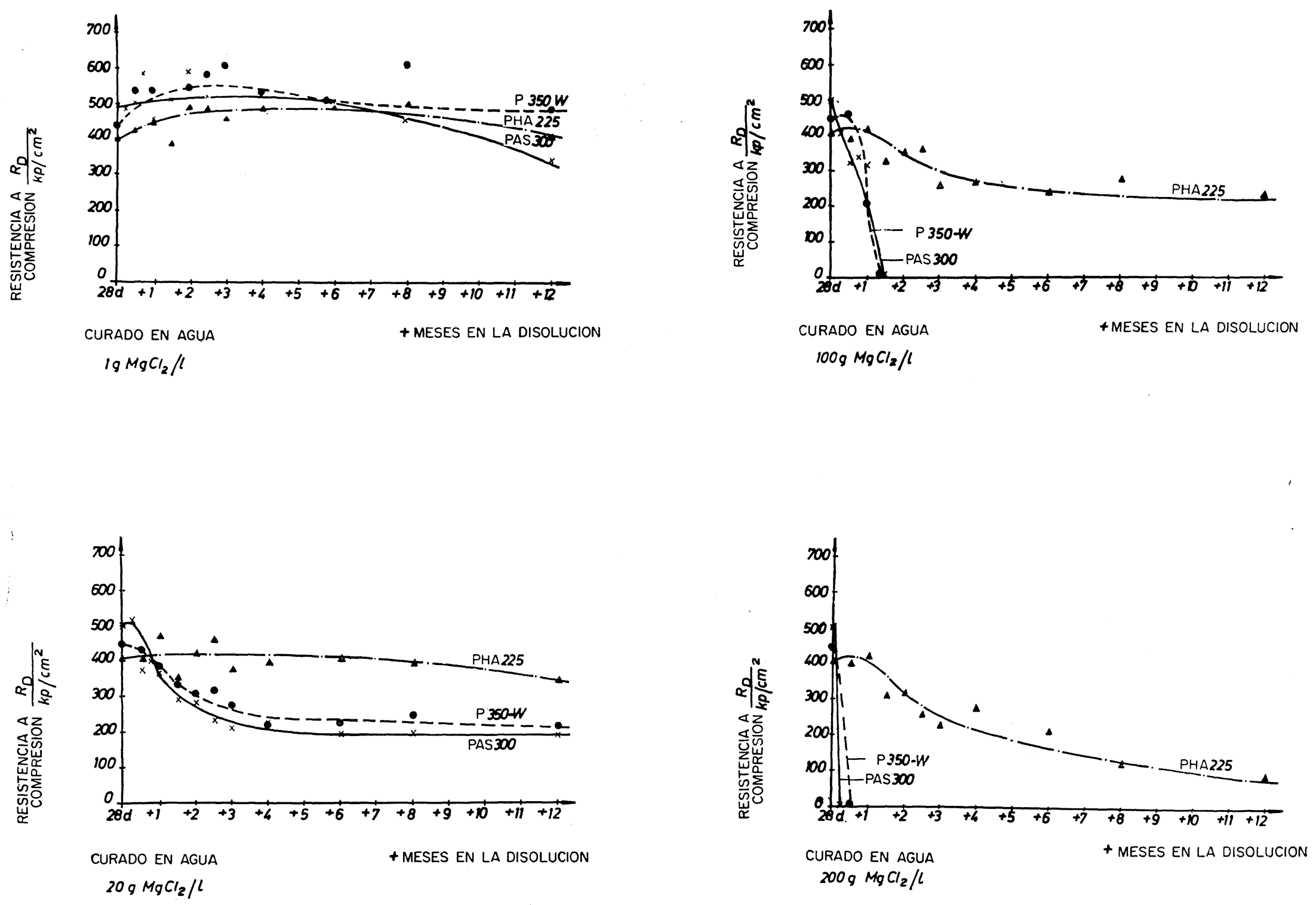

Fig. 8. - Resistencias de prismas $10 \times 10 \times 60 \mathrm{~mm}^{3}$ preparados con cementos P; PAS y $\mathrm{PHA}$ sumergidos en disoluciones de $\mathrm{MgCl}_{2}$. 


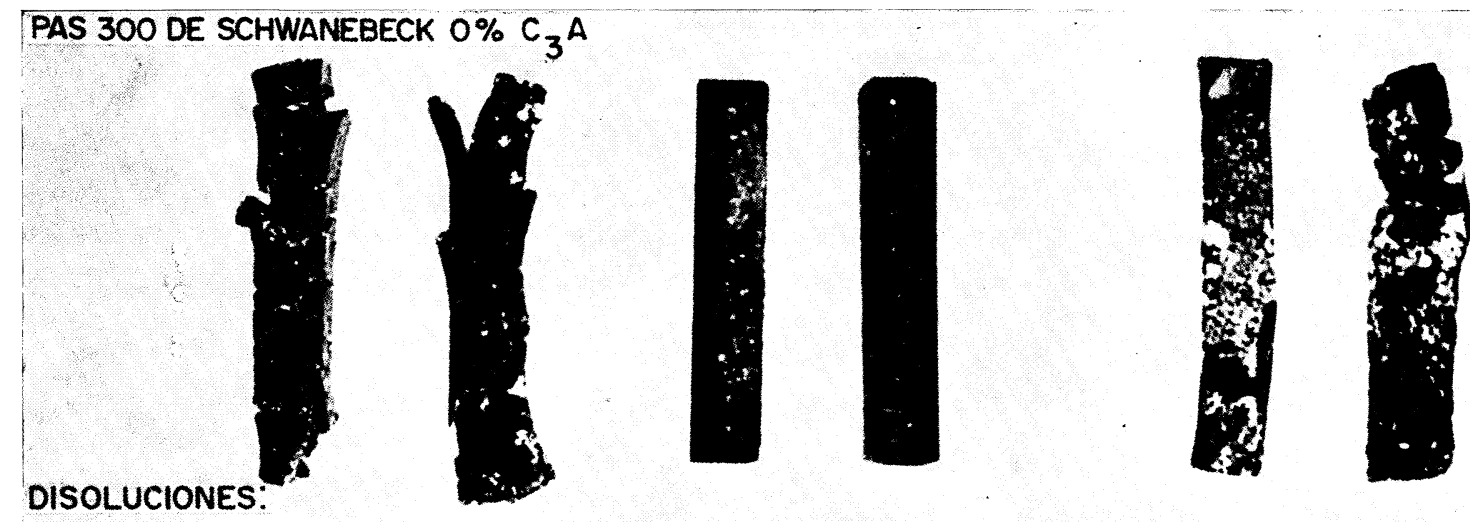

$$
100 \mathrm{~g} / \mathrm{I} \quad \mathrm{MgCl}_{2}
$$

$25,3 \mathrm{~g} / \mathrm{I} \mathrm{MgSO}_{4}$

$100 \mathrm{~g} / \mathrm{I} \mathrm{MgCl}_{2}$

PAS 300 DE SCHWANEBECK CON $6 \% C_{3}$ A AÑADIDO

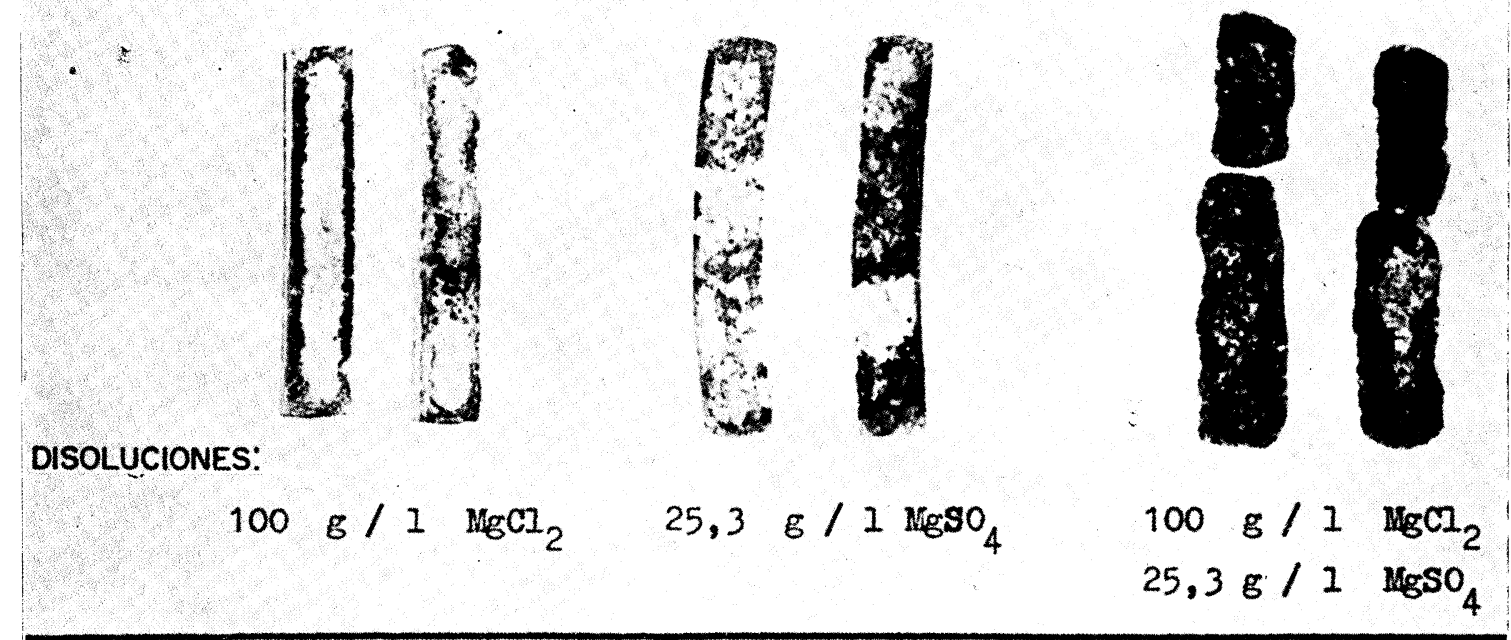

PAS 300 DE SCHWANEBECK CON $12 \% C_{3}$ A AÑADIDO

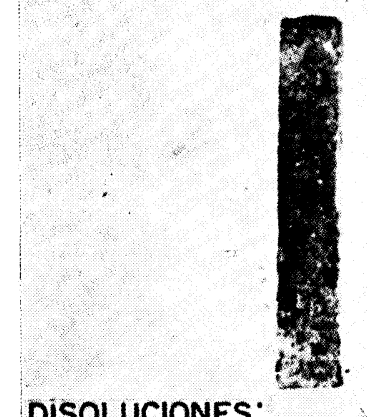

DISOLUCIONES:

$$
100 \mathrm{~g} / \mathrm{I} \quad \mathrm{MgCl}_{2}
$$
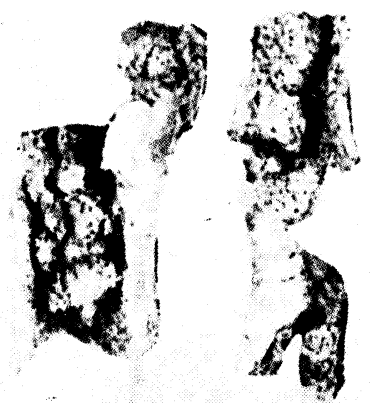

$25,3 \mathrm{~g} / \mathrm{I} \quad \mathrm{MgSO}_{4}$
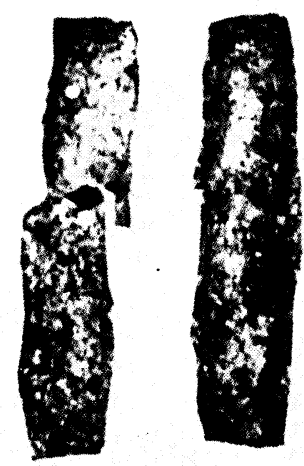

$100 \mathrm{~B} / \mathrm{I} \mathrm{MgCl}_{2}$ $25,3 \mathrm{~g} / \mathrm{I} \mathrm{MgSO}_{4}$

Fig. 11.-Influencia del $\hat{C}_{3} A$ contenido en los morteros de PAS, sobre la resistencia a corrosión por las disoluciones de $\mathrm{MgSO}_{4}$ y de $\mathrm{MgCl}_{2}$.

Estado de los prismas de $10 \times 10 \times 60 \mathrm{~mm}^{3}$ después de 6 meses en contacto con la disolución agresiva. 
El examen con rayos X acusó la presencia de la fase CSH y, en los cementos de tipo portland, la presencia también de hidróxido cálcico, pequeñas cantidades de ettringita y de yeso.

El análisis térmico diferencial puso de manifiesto grandes diferencias entre los componentes de las pastas en los prismas mantenidos en agua y los conservados en la disolución de sulfato magnésico: también se diferenciaron las zonas externas de las internas, apreciándose en las primeras la existencia de los productos de la corrosión.

Las diferencias entre la pasta de los cementos portland y PAS, sumergidos en la disolución de sulfato, son las siguientes:

- ausencia de $\mathrm{Ca}(\mathrm{OH})_{2}$ en el portland y presencia en el PAS;

- más cantidad de $\mathrm{Mg}(\mathrm{OH})_{2}$ en el portland que en el PAS;

- más cantidad de ettringita y de yeso en el portland que en el PAS;

- mucha menor cantidad de tobermorita en el portland que en el PAS;

- ausencia de fase CAFH en el portland y pequeña cantidad en el PAS ;

- igual cantidad de cemento sin hidratar en los tipos portland y PAS;

- mucha cantidad de agua absorbida en el portland y muy poca en el PAS.

Se pueden poner de manifiesto las diferencias entre portland y PAS calculando las cantidades de los componentes referidas, para cada uno de ellos, a las existentes en la pasta de los prismas conservados en agua, valorando éstas como 100. Así:

Portland: $\mathrm{CaCO}_{3}=62 \% ; \mathrm{Ca}(\mathrm{OH})_{2}=0 \% ; \mathrm{Mg}(\mathrm{OH})_{2}=333 \%$; Ettringita y yeso $=$ $=4.200 \% ;$ Tobermorita $=7,5 \% ; \mathrm{CAFH}=0 \% ;$ Agua adsorbida $=160 \%$.

PAS: $\mathrm{CaCO}_{3}=75 \% ; \mathrm{Ca}(\mathrm{OH})_{2}=73 \% ; \mathrm{Mg}(\mathrm{OH})_{2}=140 \% ;$ Ettringita y yeso $=680 \%$; Tobermorita $=100 \% ; \mathrm{CAFH}=27 \% ;$ Agua adsorbida $=40 \%$.

La disolución de sulfato magnésico ha reaccionado con el portland en gran escala como lo indican las cifras de $\mathrm{Ca}(\mathrm{OH})_{2}$ perdidas; de $\mathrm{Mg}(\mathrm{OH})_{2}$ fijadas; de ettringita y yeso formadas, y de agua adsorbida.

El cemento de horno alto, con $66 \%$ de escorias, que puede encuadrarse en el PHA del Pliego español, cuando se le aplica el criterio comparativo antes utilizado para los portland, da los números siguientes:

$\mathrm{CaCO}_{3}=62 \% ; \mathrm{Ca}(\mathrm{OH})_{2}=50 \% ; \mathrm{Mg}(\mathrm{OH})_{2}=140 \% ;$ Ettringita y yeso $=100 \%$; Tobermorita $=49 \% ; \mathrm{CAFH}=80 \% ;$ Agua adsorbida $=0 \%$.

El autor explica la mejor resistencia química del PHA debida a la fijación de $\mathrm{Ca}(\mathrm{OH})_{2}$ por las escorias (no creo cierta esta apreciación, pues las escorias básicas, granuladas, no son una puzolana; el hidróxido cálcico excita la hidraulicidad latente de la escoria, como lo hace el hidróxido sódico); a la gran cantidad de compuestos geliformes que las escorias engendran al hidratarse y que entorpecen la movilidad de los cationes-calcio por adsorción superficial y, también, limitan la formación de ettringita y de yeso.

De todo ello se deduce que no es discriminatoria la relación entre el yeso y las fases análogas a la ettringita formadas, sino la cantidad de $\mathrm{SO}_{4}{ }^{2-}$ combinado. De acuerdo con esto, Riedel construye unas gráficas que representan las variaciones de la concentración de $\mathrm{SO}_{4}{ }^{2-} ; \mathrm{Ca}^{2+}$ y $\mathrm{Mg}^{2+}$ en las disoluciones de 1,26 y de $12,6 \mathrm{~g}$ de $\mathrm{MgSO}_{4}$ producidas a lo largo de 14 meses por los tres conglomerantes: P, PAS y PH. 
Clasifica la agresividad de las disoluciones de $\mathrm{MgSO}_{4}$, según su concentración, en:

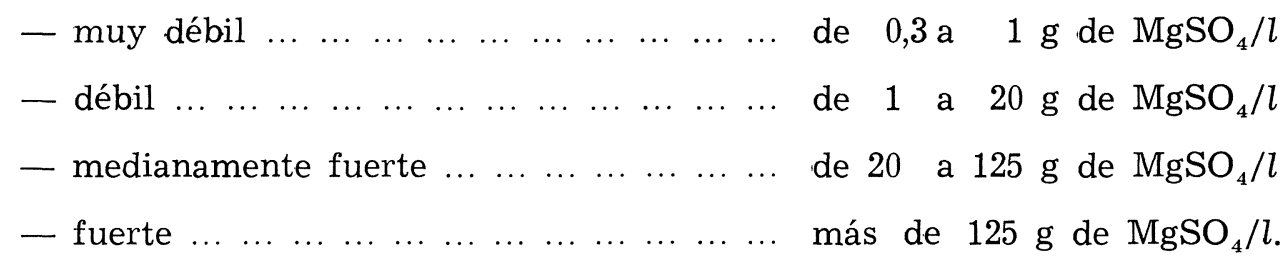

Las disoluciones débiles de $\mathrm{MgSO}_{4}$ penetran rápida y profundamente en los morteros de cemento en los que se encuentran un $\mathrm{pH}$ alto $(12,6)$ y gran cantidad de $\mathrm{Ca}(\mathrm{OH})_{2}$.

En el mortero de portland se forma ettringita y, después de combinada alguna cantidad de sulfato, precipita el $\mathrm{Mg}(\mathrm{OH})_{2}$ poco soluble; desciende el $\mathrm{pH}$, se forma yeso, sílice e hidróxido de aluminio al estado de geles.

En el mortero de PAS hay muy poca cantidad de aluminato para combinarse con el sulfato, pero el $\mathrm{C}_{4} \mathrm{AF}$ hidratado se combina y, por tanto, no es totalmente resistente.

Las disoluciones medianamente agresivas de $\mathrm{MgSO}_{4}$, también provocan la formación de ettringita, con valores altos del $\mathrm{pH}$, al entrar en contacto con los morteros de cemento portland. La mayor formación de $\mathrm{Mg}(\mathrm{OH})_{2}$ produce una disminución del $\mathrm{pH}$; los silicatos y los aluminatos cálcicos son destruidos y se forma mucho yeso.

Después de 6 mses de contacto, en el exterior de los morteros se forma yeso, así como también productos geliformes del ataque; en el interior se encuentran yeso y productos similares a la ettringita. El continuado descenso del pH incrementa la formación de yeso.

\section{Corrosión por el cloruro magnésico $\mathbf{M g C l}_{2}$}

Aunque la mayor solubilidad del cloruro magnésico permite preparar disoluciones muy concentradas, el autor emplea en sus ensayos cantidades de cloruro que se corresponden, en concentración iónica, con las utilizadas en los ensayos con $\mathrm{MgSO}_{4}$.

Las medidas mediante las frecuencias de resonancia pusieron de manifiesto un aumento inicial del módulo de elasticidad, seguido de una caída final de los valores hasta llegar a la destrucción total.

Disoluciones hasta $1 \mathrm{~g}$ de $\mathrm{MgCl}_{2}$ : A los 12 meses de contacto se aprecian diferencias de resistencia química entre los cementos ensayados. El portland y el PHA resisten al ataque. El PAS pierde, en ese lapso de tiempo, un tercio de sus resistencias mecánicas iniciales. Estas diferencias se acusan todavía más en los prismas sumergidos en disoluciones de $5 \mathrm{~g}$ de $\mathrm{MgCl}_{2} / l$.

Disoluciones hasta $50 \mathrm{~g}$ de $\mathrm{MgCl}_{2}$ : El portland y el PAS pierden rápidamente resistencias mecánicas. El PAS aparece peor que el P.

Disoluciones con más de $50 \mathrm{~g}$ de $\mathrm{MgCl}_{2}$ : Son muy agresivas para el mortero. Tanto el portland como el PAS apenas resisten. Sólo el PHA posee alguna resistencia.

Las resistencias entre los componentes hidratados de los conglomerantes y las disoluciones de cloruro dan lugar a cambios de concentraciones iónicas utilizables para juzgar la resistencia química de aquéllos.

En la figura 9 se puede apreciar, para concentraciones de $50 \mathrm{~g} \mathrm{de} \mathrm{MgCl}_{2} / l$, que los morteros de portland y de PAS experimentan una pérdida de calcio mayor y con más velocidad que el mortero de PHA. 
En las disoluciones con $10 \mathrm{~g}$ de $\mathrm{MgCl}_{2} / l$ se establece un equilibrio que estabiliza la pérdida de calcio, y para el PHA, con disoluciones más concentradas, es patente una menor fijación de ion-magnesio.

En disoluciones hasta $100 \mathrm{~g}$ de $\mathrm{MgCl}_{2} / l$ el cemento portland pierde menos calcio que el PAS.

Los ensayos con pasta pura se realizaron con prismas de $10 \times 10 \times 60 \mathrm{~mm}^{3}$ conservados durante 2 años en disolución de $350 \mathrm{~g}$ de $\mathrm{MgCl}_{2} / l$.

Sobre los prismas y en el interior de las grietas se formó un "fieltro" sólido, blanco, que, examinado con rayos $\mathrm{X}$, se identificó como $\mathrm{Mg}_{2}(\mathrm{OH})_{3} \mathrm{Cl}$.

Los prismas de portland y de PAS adquirieron una textura laminar. Las capas exteriores contenían, principalmente, oxicloruro de magnesio $(d=8,04 ; 4,02 ; 3,81)$ junto con pequeñas cantidades de $\mathrm{Mg}(\mathrm{OH})_{2}$, tricloruro y silicato cálcico hidratado. Las zonas interiores contenían, preponderantemente, sal de Friedel $(d=7,87 ; 3,99 ; 3,86)$, oxicloruro de magnesio, fase $\mathrm{M}_{2} \mathrm{~S}_{3} \mathrm{H}_{2}(d=7,4 ; 3,66 ; 2,46)$ y, además, hidróxidos cálcico y magnésico.

Los prismas de PHA no presentaban diferencias entre las capas externas y las internas. Los principales compuestos fueron la sal de Friedel y la tobermorita, junto con pequeñas cantidades de hidróxido y silicato magnésico en el exterior y tricloruro en el núcleo.

La relativa buena resistencia a la corrosión del portland con $\mathrm{C}_{3} \mathrm{~A}$ hasta 10 y $14 \%$ frente a las disoluciones de $\mathrm{MgCl}_{2}$ debe atribuirse a la formación de sal de Friedel. Los portland pobres o exentos de $\mathrm{C}_{3} \mathrm{~A}$ (PAS), aparecen más débiles que en el portland normal.

Las disoluciones de cloruro magnésico son tanto más agresivas cuánto más disponibilidades de ion magnesio posean. Riedel establece una escala de agresividad, que es la que a continuación figura:

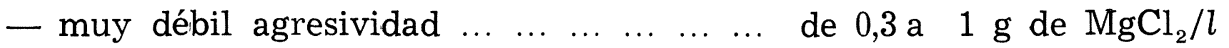

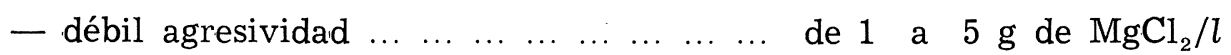

- agresividad media $\ldots \ldots \ldots \ldots \ldots \ldots \ldots$ de 5 a 50 g de $\mathrm{MgCl}_{2} / l$

- fuerte agresividad $\ldots \ldots \ldots \ldots \ldots \ldots \ldots \ldots$ más de $50 \mathrm{~g}$ de $\mathrm{MgCl}_{2} / l$.

Cuando la disolución penetra en el mortero de cemento portland destruye rápidamente el $\mathrm{C}_{3} \mathrm{~S}$ y el $\mathrm{C}_{4} \mathrm{AF}$ hidratados; el $\mathrm{C}_{3} \mathrm{~A}$ forma monocloruro (Sal de Friedel) que contiene la corrosión. Por esta razón los cementos PAS resisten menos frente al $\mathrm{MgCl}_{2}$ que el portland.

El autor, Sr. Riedel, finaliza el trabajo con los resultados de las experiencias llevadas a cabo con prismas de un PAS y del mismo con adiciones de 6 y $12 \%$ de $\mathrm{C}_{3} \mathrm{~A}$, mantenidos durante 6 meses en disoluciones agresivas de sulfato y cloruro magnésicos. Frente al $\mathrm{MgSO}_{4}$ no resistió el cemento que contenía 10 a $12 \%$ de $\mathrm{C}_{3} \mathrm{~A}$; el que contenía $6 \%$ mostró una resistencia media, y alta resistencia el PAS sin $\mathrm{C}_{3} \mathrm{~A}$. Frente al $\mathrm{MgCl}_{2}$, la resistencia crece a medida que es mayor el contenido de $\mathrm{C}_{3} \mathrm{~A}$. 